\title{
Descomposición y calidad físico-química foliar de 24 especies dominantes de los pastizales de altura de las sierras de Córdoba, Argentina
}

\author{
María Poca ${ }^{1,2, 凶}$; Natalia Pérez-Harguindegur ${ }^{1,2}$; María V. Vaieretti ${ }^{1} \&$ Ana M. Cingolani ${ }^{1,2}$ \\ 1. Instituto Multidisciplinario de Biología Vegetal. 2. Facultad de Ciencias Exactas, Físicas y Naturales CONICET- \\ Universidad Nacional de Córdoba, Argentina.
}

\begin{abstract}
Resumen. Tanto la calidad química de la broza como las características físicas de las hojas verdes han sido indicadas como determinantes de la descomposición de la broza. El objetivo de este trabajo fue analizar la relación entre la descomposición, la calidad química de la broza y la calidad física de las hojas verdes de 24 especies dominantes de los pastizales mésicos y húmedos de las sierras altas de Córdoba (Argentina). Además, comparamos el patrón de asociación entre esas características con el descripto para las especies de las sierras bajas de la misma región. Medimos la descomposición de la broza mediante la incubación en jardín común en dos periodos (70 y 196 días). Como indicadores de la calidad química de la broza medimos el contenido de componentes lábiles (nitrógeno) y el de componentes recalcitrantes (celulosa, hemicelulosa y lignina). A partir de estos indicadores calculamos el contenido de fibras (sumas de compuestos recalcitrantes), la proporción holocelulosa/fibras y las relaciones lignina:nitrógeno, holocelulosa:nitrógeno y fibras:nitrógeno. Como indicador de calidad física de las hojas verdes medimos el área foliar específica. La descomposición aumentó a medida que disminuyó la relación fibras:nitrógeno para ambos periodos. Al contrario de lo encontrado en otros trabajos, el contenido de lignina y el área foliar específica no se correlacionaron significativamente con la descomposición. En cambio, las relaciones entre descomposición y calidad resultaron similares a las documentadas para las especies dominantes de las sierras bajas de Córdoba. Esta similitud, a pesar de las diferencias en composición de especies, tipos funcionales y regímenes climáticos, indica una consistencia del control químico en la descomposición.
\end{abstract}

[Palabras clave: descomposición foliar, calidad foliar, calidad de la broza, procesos ecosistémicos]

\begin{abstract}
Aвstract. Decomposition and physico-chemical foliar traits of 24 dominant species of highland grasslands in the Córdoba Mountains, Argentina: Litters' chemical and green leaves' physical quality have been indicated as determinants of litter decomposition. The objective of this work was to analyze the relationship between litter decomposition and chemical quality and green leaves physical quality of 24 dominant species of mesic and humid highland grasslands, Córdoba, Argentina. In addition, we compared the pattern of association between these variables with that described for the lowland mountains of the same region. We measured litter decomposition through a common garden experiment with two retrieval dates (70 and 196 days). We measured labile compounds (nitrogen) and recalcitrant compounds (cellulose, hemicelluloses and lignin) as litter chemical quality indicators. Besides, we calculated from these indicators the fiber content (sum of recalcitrant compounds), the holocellulose/fiber proportion and the lignin:nitrogen, holocellulose:nitrogen and fiber:nitrogen ratios. We measured the specific leaf area as a physical quality indicator of the green leaves. The decomposition increased mainly while the fiber:nitrogen ratio decreased for both retrieval dates. Contrary to other works, the lignin content and the specific leaf area were not significantly associated to the decomposition. The relationships that we found in this work between decomposition and quality parameters are similar to the ones described by Vaieretti et al. (2005) for the species of the lowland mountains of Córdoba. This similitude, in spite of the differences in species composition, functional types and environmental variables, indicate a consistent chemical control over decomposition.
\end{abstract}

[Keywords: foliar decomposition, foliar quality, litter quality, ecosystem processes]

\section{INTRODUCCIÓN}

La descomposición es el proceso ecosistémico que permite que los nutrientes de la biomasa se tornen disponibles para ser absorbidos por las plantas o por los microorganismos (Cornelissen et al. 1999; Chapin et al. 2002; Vivanco \& Austin 2006). Por esta razón, es un proceso fundamental en la regulación del reciclado de la materia y la energía. La descomposición depende de tres factores:

$\bowtie$ pocamaria@gmail.com las condiciones ambientales (temperatura y humedad), la calidad del material vegetal en descomposición y las características de la comunidad de descomponedores (Swift et al. 1979). De estos factores, la calidad del material vegetal juega un rol determinante en la tasa de descomposición a escala local ya que a esta escala se considera que las condiciones ambientales permanecen relativamente constantes (Lavelle et al. 1993; Aerts 1997; Kazakou et al. 2009; Cornwell et al. 2008). 
Numerosos trabajos han demostrado que la descomposición de la broza (o material senescente) se relaciona con diversos parámetros indicadores de su calidad química, tales como la relación carbono:nitrógeno, el contenido de celulosa, hemicelulosa y lignina (Cadish \& Giller 1997; Pérez Harguindeguy et al. 2000a,b; Garnier et al. 2004; Vaieretti et al. 2005; Vivanco \& Austin 2006; Cortez et al. 2007; Cornwell et al. 2008). Además, se ha propuesto que las características químicas y físicas que hacen que la broza sea más o menos descomponible son la herencia de los atributos funcionales de las hojas verdes (e.g., el contenido de nitrógeno, el área foliar específica y la dureza foliar, entre otros) (Pérez Harguindeguy et al. 2000a,b; Vaieretti et al. 2005; Cortez et al. 2007; Garnier et al. 2004; Cornwell et al. 2008).

Para el centro de Argentina se ha encontrado que tanto los caracteres químicos de la broza, como los físicos y químicos de las hojas verdes son buenos predictores de la descomposición de la broza de las especies que constituyen la flora (Cornelissen et al. 1999; Pérez Harguindeguy et al. 2000a,b; Vaieretti et al. 2005). En un análisis de la flora dominante a lo largo de un gradiente regional de precipitación del centrooeste de Argentina, Pérez Harguindeguy et al. $(2000 a, b)$ encontraron que la relación carbono: nitrógeno de la broza y la dureza foliar de las hojas verdes se relacionaron de manera estrecha y negativa con la descomposición de la broza de las especies estudiadas. Vaieretti et al. (2005), en una escala local y en un análisis de la flora de la parte baja de las sierras de Córdoba, encontraron que la descomposición de las especies se asoció de manera negativa con el contenido de fibras de su broza (suma del contenido de celulosa, hemicelulosa y lignina) y de forma positiva con el área foliar específica de las hojas verdes. El contenido de nitrógeno de las hojas verdes también estuvo asociado positivamente a la descomposición de estas especies, aunque de manera más laxa.

En este trabajo analizamos la relación entre la descomposición y la calidad química de la broza y la calidad física de las hojas verdes de 24 especies dominantes de los pastizales mésicos y húmedos del piso superior de las sierras de Córdoba, Argentina (por encima de 1700 m.s.n.m.). Además, discutimos si los controles de la descomposición encontrados en esta parte de las sierras son similares a lo obtenido por Vaieretti et al. (2005) para la flora de las parte baja de las sierras de Córdoba (a 1000 m.s.n.m.).

\section{MAteriales y MÉTOdOS}

\section{Área de estudio}

Realizamos el trabajo en la Pampa de Achala, ubicada en el piso superior de las sierras de Córdoba

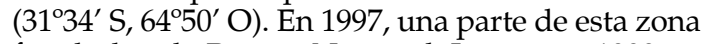
fue declarada Parque Nacional. Luego, en 1999, se extendió el área de conservación y se creó la Reserva Hídrica Provincial de Achala. A 2200 m.s.n.m., altitud aproximada de nuestro sitio de estudio, la temperatura media del mes más frío (julio) es 5.8 ${ }^{\circ} \mathrm{C}$, mientras que la del mes más cálido (diciembre) es $11^{\circ} \mathrm{C}$. La temperatura media anual es $8.5^{\circ} \mathrm{C}$. No hay meses libres de heladas. La precipitación media anual del área es $900 \mathrm{~mm}$, concentrados entre los meses de octubre y abril (Cabido \& Acosta 1985; Colladon et al. 2010). Los suelos son molisoles derivados de rocas graníticas y partículas de textura fina consecuencia de la erosión eólica del Cuaternario (Cabido et al. 1987). El paisaje está conformado por distintas geoformas que incluyen fondos de valle, quebradas y planicies con diferente grado de disección. Según la condición topográfica y el disturbio pasado y presente, la vegetación de la región consiste en un mosaico de bosques de Polylepis australis BITT y pastizales (Cingolani et al. 2004, 2008). Los pastizales pueden clasificarse en xéricos, mésicos y húmedos según las especies dominantes y la disponibilidad de agua en el suelo, que a su vez se asocia a la posición topográfica (Cingolani et al. 2003). En los tres tipos de ambiente se pueden distinguir parches de vegetación con diferentes fisonomías asociadas a distintos grados de pastoreo: céspedes, pajonales abiertos y pajonales cerrados. El sitio de estudio abarcó pastizales mésicos y húmedos, donde los céspedes están dominados por especies de dicotiledóneas herbáceas y graminoides como Lachemilla pinnata (Ruiz \& Pav.) Rothm., Carex fuscula ssp. fuscula d'Urv. y Eleocharis pseudoalbibracteata S. González \& Guagl., mientras que los pajonales cerrados están dominados por gramíneas en mata como Poa stuckertii (Hack.) Parodi y Deyeuxia hieronymi (Hack.) Türpe. Los pajonales abiertos representan una situación intermedia entre las dos fisonomías anteriores, siendo Deyeuxia hieronymi la principal gramínea en mata en ellos (Cingolani et al. 2003; Vaieretti et al. 2010). A su vez, estas tres fisonomías se diferencian en las características físicas y químicas de las hojas de las especies dominantes y, en consecuencia, también de la broza de las mismas (Díaz et al. 1994; Pucheta et al. 1998a,b; Cingolani et al. 2007). Los céspedes, donde los herbívoros tienden a congregarse, se caracterizan por poseer especies más palatables, con mayor contenido de nutrientes y características foliares indicadoras de altas tasas de crecimiento relativo. En el otro extremo están los pajonales cerrados, dominados por gramíneas en mata y poco utilizados por los herbívoros (Pucheta et al. 1998a; Cingolani et al. 2003, 2007).

\section{Selección de especies}

Sobre la base de un relevamiento florístico realizado por Vaieretti et al. (2010) en la zona de 
estudio, elegimos 24 especies dominantes. Para ello seleccionamos de la lista de especies de cada censo aquellas que sumaban un $80 \%$ de cobertura relativa. Luego confeccionamos una nueva lista de especies con todas las que quedaron seleccionadas para cada censo, y esas son las especies que fueron determinadas como dominantes (siguiendo a Garnier et al. 2004; Pakeman \& Quested 2007; Pérez Harguindeguy et al. 2013; Información Suplementaria Tabla S1). Tres de las 24 especies seleccionadas fueron gramíneas de crecimiento en mata, 14 fueron graminoides (gramíneas bajas y otras monocotiledóneas graminiformes) y siete fueron dicotiledóneas herbáceas (Tabla S1). La nomenclatura de las especies sigue a Zuloaga et al. (2008) y a su actualización on-line (www.darwin.edu.ar).

\section{Descomposición de las especies}

Para determinar la descomposición de las especies seleccionadas utilizamos la técnica de bolsas de broza (Cornelissen 1996; Berg \& Laskowski 2006; Pérez Harguindeguy et al. 2013). Preparamos 20 bolsas (réplicas) por especie, cada una con $\sim 1 \mathrm{~g}$ de broza secada al aire. Para cada réplica por especie recolectamos hojas senescentes, sin descomponer (Cornelissen 1996; Cornelissen \& Thompson 1997) de un individuo (o grupo de individuos agrupados espacialmente, para el caso de plantas pequeñas). La broza la recolectamos del suelo, exceptuando algunas especies como Eleocharis pseudoalbibracteata, cuyas hojas senescentes quedan en pie. Realizamos la recolección durante los meses de septiembreoctubre del año 2008 por ser el período de máxima acumulación de biomasa senescente en la zona de estudio (Vaieretti 2010).

Para la construcción de las bolsas utilizamos una malla de nylon de $0.3 \mathrm{~mm}$. Si bien esta malla no permite el paso de invertebrados, se considera que el aporte de éstos a la tasa de descomposición es pequeño en comparación a la actividad de protozoos, bacterias y hongos (Cornelissen 1996; Perez Harguindeguy et al. 2000b; Vaieretti et al. 2010). Dentro de nuestra misma área de estudio, Vaieretti et al. (2010) no encontraron diferencias en la descomposición de materiales estándar (papel de filtro y hojas senescentes de Poa stuckertii) incubados en bolsas de nylon de 0.3 y $1 \mathrm{~mm}$ de malla.

La descomposición de la broza de las distintas especies se determinó mediante su incubación en una cama de descomposición (experimento dejardín común). Al incubar las bolsas de descomposición de manera simultánea bajo las mismas condiciones ambientales, la pérdida de peso seco resultante es un reflejo de los aspectos estructurales y químicos de la broza, y constituye una expresión de su calidad para los microorganismos (Cornelissen 1996; Cornelissen et al. 1999; Pérez Harguindeguy et al. 2013). Para la construcción de la cama de descomposición, dentro de la zona de estudio en un potrero sin pastoreo seleccionamos al azar un sector de $\sim 16 \mathrm{~m}^{2}(4 \times 4 \mathrm{~m})$ del que extrajimos todas las plantas y las raíces más importantes. Sobre el área limpia de vegetación colocamos al azar las bolsas de broza, con una cara en contacto con el suelo, y las cubrimos con una fina capa de broza del área para simular condiciones naturales de descomposición. El experimento se inició en la estación de verano, que es la época más húmeda y más cálida en la región. Recolectamos la mitad de las bolsas (10 réplicas/especie) luego de 70 días de incubación (desde diciembre de 2008 a marzo de 2009) y las bolsas restantes (10 réplicas/especie) luego de 196 días de incubación (desde diciembre de 2008 a julio de 2009). El tiempo de incubación se determinó sobre la base de estudios previos realizados en la región (Vaieretti et al. 2005, 2010, 2013) que indican que en estos lapsos se pueden detectar diferencias en la descomposición entre especies sin llegar al 100\% de la pérdida del material de ninguna muestra.

Después de ser recolectadas, las bolsas de broza fueron almacenadas a $-14{ }^{\circ} \mathrm{C}$ hasta su procesamiento. Posteriormente, las descongelamos al aire y removimos con un pincel todo material externo que pudiera haber quedado adherido a la broza. A continuación, secamos las muestras a 70 ${ }^{\circ} \mathrm{C}$ durante al menos $48 \mathrm{~h}$. Finalmente, calculamos la pérdida de peso seco (\%) en relación al peso seco inicial antes de la incubación ((1-(peso seco final/ peso seco inicial)) $\times 100$ ). Para corregir el peso seco inicial debido a la humedad que pueda tener la broza secada al aire, pesamos sub-muestras de tres réplicas/especie y luego las secamos en la estufa a $70^{\circ} \mathrm{C}$ al menos por $48 \mathrm{~h}$. Las sub-muestras fueron secadas en estufa y el valor obtenido fue restado al peso inicial de la broza; así se obtuvo un promedio del contenido de humedad de cada especie. Este valor fue restado a todos los pesos iniciales de broza de las bolsas.

\section{Calidad química inicial de la broza}

Como indicadores de la calidad química de la broza utilizamos el contenido de celulosa, hemicelulosa, lignina y nitrógeno de las especies seleccionadas. Para ello empleamos tres muestras de broza $(n=3)$ recolectadas en el campo del mismo modo que colectamos las muestras para realizar las bolsas de descomposición. Cada muestra tenía $\sim 5$ $\mathrm{g}$ de material. Secamos la broza de cada muestra en estufa a $60{ }^{\circ} \mathrm{C}$ por $48 \mathrm{~h}$ y luego la molimos y homogeneizamos. Determinamos el contenido de celulosa, hemicelulosa y lignina siguiendo la técnica de Goering \& Van Soest (1970) y el contenido de nitrógeno utilizando un Autoanalizador (RFA 300-Alpken, Wilsonville, O.R., USA) siguiendo la técnica propuesta por O'Neill \& Webb (1970). Estas determinaciones se realizaron en el Laboratorio de Evaluación de Forrajes, INTA E.E.A. Bariloche. A partir de los parámetros químicos medidos calculamos las siguientes variables: el contenido de holocelulosa (suma del contenido de celulosa y hemicelulosa), el contenido total de fibras (suma del contenido de lignina, celulosa y hemicelulosa; de aquí en más "fibras"), la proporción holocelulosa/ fibras, la relación lignina:nitrógeno, la relación 
holocelulosa:nitrógeno y la relación fibras: nitrógeno. Algunas de estas relaciones entre variables químicas han demostrado ser mejores predictoras de la descomposición que las variables químicas aisladas (Berg et al. 1984; Cortez et al. 1996; Vaieretti et al. 2005).

\section{Calidad física del material vegetal verde}

Como medida de la calidad física del material vegetal verde utilizamos el área foliar específica $\left(\mathrm{mm}^{2} / \mathrm{mg}\right)$. En el presente trabajo determinamos el área foliar específica de cinco especies siguiendo protocolos estándares (Pérez Harguindeguy et al. 2013), mientras que para las 19 especies restantes los datos fueron obtenidos de Díaz et al. 2004 y Cingolani et al. 2007, quienes utilizaron los mismos protocolos (Información SuplementariaTabla S2). Para calcular el área foliar específica escaneamos hojas verdes frescas, totalmente expandidas y con el programa Image J determinamos el área de las mismas. Luego, secamos las hojas en la estufa a 60 ${ }^{\circ} \mathrm{C}$ por $48 \mathrm{~h}$. A partir de la división del área foliar y del peso seco de las hojas calculamos el área foliar específica. Para las especies aquí medidas consideramos 6 réplicas/especie.

\section{Análisis de datos}

Realizamos un análisis de correlación de Spearman entre las variables de calidad química de la broza (celulosa, hemicelulosa, lignina, nitrógeno, holocelulosa, fibras, holocelulosa:fibras, lignina:nitrógeno, holocelulosa:nitrógeno y fibras: nitrógeno), la calidad física de las hojas verdes (área foliar específica) y la descomposición de las 24 especies para ambos períodos de incubación (70 y 196 días). Para los análisis estadísticos utilizamos el programa estadístico InfoStat versión 2013 (Di Rienzo et al. 2013).

\section{Resultados}

Los valores de descomposición (\% de pérdida de peso seco) obtenidos luego de 70 días de incubación variaron entre $4 \%$ y $23 \%$, mientras que los valores obtenidos luego de 196 días de incubación variaron entre 10\% y $42 \%$. Las especies que se descompusieron más rápido (mayor pérdida de peso seco) fueron la graminoide Eleocharis pseudoalbibracteata y la dicotiledónea herbácea Bidens andicola var. decomposita Kunth luego de 70 y 196 días de incubación, respectivamente. Por su parte, la gramínea en mata Poa stuckertii fue la especie que se descompuso más lento durante ambos periodos de incubación (Figura 1 a y b). El patrón de descomposición de las especies estuvo altamente correlacionado entre los dos periodos de incubación $(r=0.90, P<0.001)$.

En general, el contenido de celulosa, hemicelulosa, holocelulosa y fibras fue menor
Tabla 1. Coeficientes de correlación de Spearman $(r)$ entre los caracteres químicos y físicos y la descomposición (\% de pérdida de peso seco) de las especies de la parte alta de las sierras de Córdoba luego de 70 y 196 días de incubación de la broza (primera y segunda columna). A modo comparativo, en la tercera y cuarta columna, se muestran los coeficientes de correlación de Spearman $(r)$ entre los caracteres químicos y físicos y la descomposición de las especies de la parte baja de las sierras de Córdoba luego de 69 y 139 días de incubación de la broza obtenidos por Vaieretti et al. (2005). ${ }^{*} P<0.05$; ${ }^{* *} P<0.01 ;{ }^{* * *} P<0.001$.

Table 1. Spearman's rank correlation coefficients $(r)$ between chemical and physical traits and decomposition (\% of dry mass loss) of the species of the high mountains of Córdoba after 70 and 196 days of litter incubation (first and second column). To compare, in the third and fourth, shown Spearman's Rank correlation coefficients $(r)$ between the chemical and physical traits and decomposition of the species of the low mountains of Córdoba after 69 and 139 days of litter incubation obtained by Vaieretti et al. (2005). ${ }^{*} P<0.05 ;{ }^{* *} P<0.01 ;{ }^{* * *} P<0.001$

\begin{tabular}{|c|c|c|c|c|}
\hline \multirow[b]{2}{*}{ Días de incubación } & \multicolumn{2}{|c|}{$\begin{array}{c}\text { Este trabajo } \\
\text { (sierras altas de } \\
\text { Córdoba) }\end{array}$} & \multicolumn{2}{|c|}{$\begin{array}{c}\text { Vaieretti et al. } \\
(2005) \\
\text { (sierras bajas de } \\
\text { Córdoba) }\end{array}$} \\
\hline & 70 & 196 & 69 & 139 \\
\hline Celulosa & $-0.59 * *$ & -0.6 & -0.40 & \\
\hline & $-0.53^{* *}$ & & -0.32 & \\
\hline & 0.34 & 0.3 & -0.36 & -0 \\
\hline & $-0.65^{* * *}$ & $-0.63^{* * *}$ & -0.42 & -0.44 \\
\hline Fibr & $-0.74^{* * *}$ & $-0.73^{* * *}$ & $-0.81^{* * *}$ & $-0.81^{* * *}$ \\
\hline Hol & $-0.45^{*}$ & $-0.49^{*}$ & 0.07 & 0.04 \\
\hline Nitró & $0.74^{* * *}$ & $0.67^{* * *}$ & $0.68^{* * *}$ & $0.53^{*}$ \\
\hline Lign & -0.39 & -0.30 & $-0.69^{* * *}$ & $-0.60^{* *}$ \\
\hline Hol & $-0.77^{* * *}$ & $-0.73^{* * *}$ & $-0.68^{* * *}$ & $-0.57^{* *}$ \\
\hline Fibr & $-0.81^{* * *}$ & $-0.74^{* * *}$ & $-0.85^{* * *}$ & $-0.74^{* * *}$ \\
\hline Área foliar específica & 0.31 & 0.32 & 0.27 & 0.31 \\
\hline
\end{tabular}

Nota: A partir de los datos de Vaieretti et al. (2005) se calcularon las relaciones entre variables que se consideraron en este trabajo (holocelulosa:fibras, lignina: nitrógeno, holocelulosa:nitrógeno, fibras:nitrógeno).

${ }^{\S}$ En el caso de Vaieretti et al. (2005), el contenido de nitrógeno fue determinado en hojas verdes.

en las dicotiledóneas herbáceas, tales como Bidens andicola y Lachemilla pinnata, y mayor en las gramíneas en mata, como Festuca lilloi Hack. o Poa stuckertii, y en las graminoides como Chascolytrum subaristatum (Lam.) Desv. La lignina y el nitrógeno presentaron el patrón opuesto (Tablas S2 y S3). La proporción holocelulosa:fibras, la relación holocelulosa: nitrógeno y la relación fibras:nitrógeno también mostraron valores menores en dicotiledóneas herbáceas, tales como Bidens andicola y Glandularia dissecta, y valores mayores en varias especies de gramíneas en mata y graminoides, como Deyeuxia hieronymi, Festuca lilloi, Poa hubbardiana Parodi y Juncus uruguensis Griseb. A su vez, las tres gramíneas en mata (Poa stuckertii, Deyeuxia hieronymi y Festuca lilloi) presentaron una relación lignina: nitrógeno mayor que ciertas graminoides como Sporobolus indicus (L.) R. Br. var. indicus 

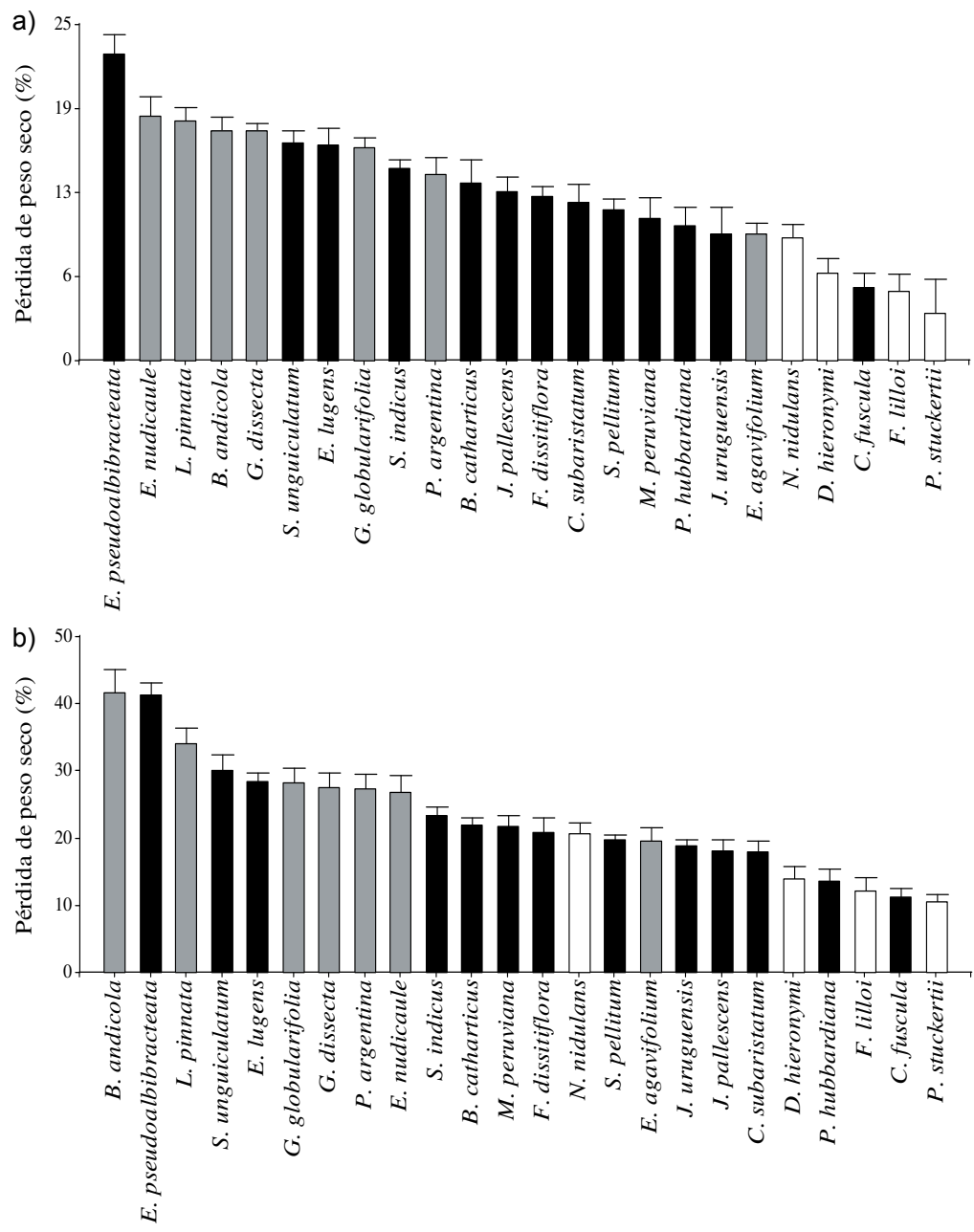

Figura 1. Porcentaje de la pérdida de peso seco (descomposición) de la broza de las 24 especies dominantes de los pastizales mésicos y húmedos de la parte alta de las sierras de Córdoba luego de: (a) 70 días de incubación y (b) 196 días de incubación. Las barras indican el valor promedio por especie con su error estándar $(n=10)$. Diferentes colores de barras indican diferentes formas de vida: en blanco las gramíneas en mata, en negro las graminoides y en gris las dicotiledóneas. Notar la diferencia de escalas entre gráficos 1a y $1 b$.

Figure 1. Percentage of dry mass loss (decomposition) of litter of the 24 dominant species of the mesic and humid grasslands of the high mountains of Córdoba after: (a) 70 days of incubation and (b) 196 days of incubation. Bars indicate mean values per specie with the standard error $(n=10)$. Different bar colors represent different life forms: white are tussock species, black are graminoids and grey are dicots. Note difference in scales between graphs $1 \mathrm{a}$ y $1 \mathrm{~b}$.

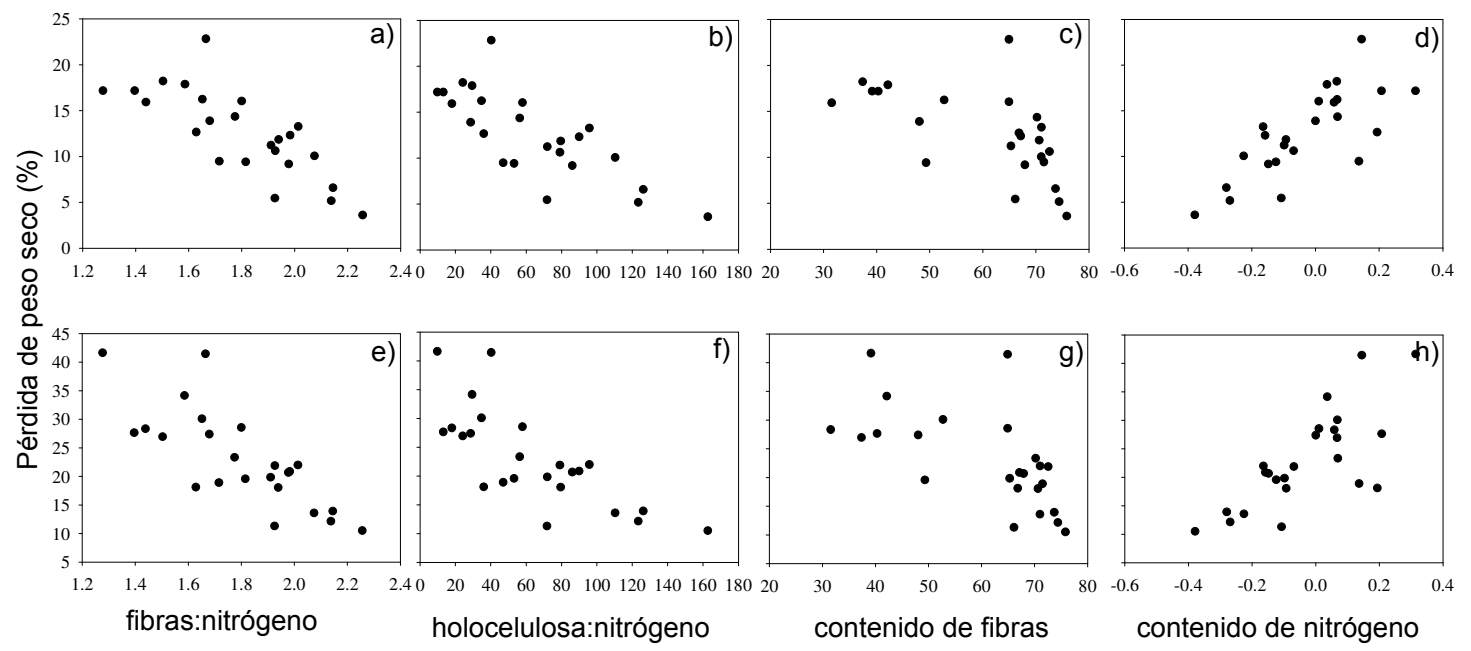

Figura 2. Relación entre la descomposición (porcentaje de pérdida de peso seco) de la broza de las 24 especies dominantes de los pastizales mésicos y húmedos de las parte alta de las sierras de Córdoba y la relación fibras:nitrógeno, la relación holocelulosa:nitrógeno, el contenido de fibras y el contenido de nitrógeno, luego de 70 (a-d) y 196 días de incubación de la broza (e-h).

Figure 2. Relationship between decomposition (\% of dry mass loss) of the 24 dominant species of the mesic and humid grasslands of the high mountains of Córdoba and the fiber:nitrogen ratio, holocelulose:nitrogen ratio, fiber content and nitrogen content, after 70 (a-d) and 196 days of litter incubation (e-h). 
y Juncus uruguensis, entre otras (Tabla S3). El área foliar específica siguió el patrón opuesto a la relación lignina:nitrógeno y varió entre 6 y $27 \mathrm{~mm}^{2} / \mathrm{mg}$ (Tabla S2).

La descomposición se correlacionó significativa y positivamente con el contenido de nitrógeno y negativamente con el contenido de fibras, la relación fibras:nitrógeno, y la relación holocelulosa: nitrógeno (Tabla 1, Figura 2). Además, la descomposición se correlacionó de forma negativa con los contenidos de celulosa, hemicelulosa, holocelulosa y la proporción de holocelulosa:fibras, pero de manera más laxa. Por otro lado, la descomposición no estuvo correlacionada ni con el contenido de lignina, ni con la relación lignina:nitrógeno ni con el área foliar específica para ninguno de los períodos analizados (Tabla 1).

\section{DisCUSIÓN}

Patrones de asociación entre la descomposición y las características de la broza y de las hojas verdes de las sierras altas de Córdoba

Los resultados obtenidos en este trabajo indican que la descomposición de la broza de las especies dominantes de los pastizales mésicos y húmedos de la parte alta de las sierras de Córdoba está fuertemente relacionada a la calidad química de la broza de las mismas, tal como se reporta en trabajos realizados en otras floras (Melillo et al. 1982; Taylor 1989; Cornelissen et al. 1999; Pérez Harguindeguy et al. 2000a,b; Vaieretti et al. 2005; Cornwell et al. 2008). En particular, las relaciones fibras:nitrógeno, holocelulosa: nitrógeno, el contenido de fibras y el contenido de nitrógeno fueron las variables que se asociaron de manera más estrecha con la descomposición de las especies dominantes de estos pastizales.

Berg (1986) y Palm \& Rowland (1997) propusieron que los compuestos lábiles (e.g., nitrógeno) suelen controlar la pérdida de peso seco durante la primera fase de la descomposición, mientras que los compuestos recalcitrantes (e.g., lignina o carbohidratos lignificados) controlan la segunda fase. En numerosos trabajos ha sido corroborado que tanto el contenido de nitrógeno y de lignina, como la relación entre ambos, son buenos predictores de la descomposición de la broza (Melillo et al. 1982; Taylor et al. 1989; Aerts 1997; Cornwell et al. 2008; Zhang et al. 2008). En general, las especies con mayor contenido inicial de nitrógeno, menor contenido de lignina, o baja relación lignina:nitrógeno presentan mayores tasas de descomposición en relación a especies que tengan los atributos opuestos. A la vez, el contenido inicial de nitrógeno domina las primeras etapas de la descomposición, mientras que la lignina adquiere importancia en etapas posteriores (Meentemeyer 1978; Swift et al. 1979; Melillo et al. 1982; Berg et al. 1984; Berg et al. 1986). Sin embargo, la mayoría de estos patrones han sido probados sobre floras de bosques templados, sistemas mediterráneos y bosques tropicales (Melillo et al. 1982; Taylor et al. 1989; Cortez et al. 1996; Loranger et al. 2002); la información sobre bosques subtropicales y sistemas sub-húmedos es, sin embargo, mucho más escasa.

Para las especies dominantes de los pastizales de la parte alta de las sierras de Córdoba, la descomposición aumentó significativamente con el aumento en la concentración de nitrógeno de la broza para los dos períodos de incubación analizados. Además, el nitrógeno presentó una asociación positiva con el contenido de lignina. Contrariamente a lo reportado por otros trabajos (Taylor et al. 1989), las especies dicotiledóneas de nuestro sitio de estudio presentaron un alto contenido de nitrógeno y también de lignina, mientras que las gramíneas presentaron el patrón opuesto (Tablas S2 y S3). Al mismo tiempo, la lignina no estuvo asociada significativamente a la descomposición. Esto puede deberse a que cuando el contenido de lignina es muy bajo (en relación al contenido de holocelulosa) no influye sobre la tasa de descomposición, y cuando el contenido de lignina es alto sigue siendo bajo en relación al contenido total de fibras. Entonces, en nuestro trabajo el contenido de fibras es el mejor indicador de los componentes recalcitrantes, más que la lignina en sí misma. Por otro lado, en nuestro estudio, ninguna de las especies seleccionadas superó el 50\% de pérdida de peso seco luego de 196 días de incubación. Por lo tanto, no podemos descartar que el proceso de descomposición se encontrara en etapas tempranas o intermedias, en las cuales todavía dominan los compuestos lábiles como el nitrógeno. En los pastizales de altura aquí analizados las etapas tardías de la descomposición, a las cuales se refieren Berg (1986) y Palm \& Rowland (1997), podrían alcanzarse luego de un año o más de incubación debido a las bajas temperaturas durante la noche y en invierno y a la falta de humedad durante la estación invernal (Vaieretti et al. datos no publicados). 
En casos como este, en los que la descomposición se encuentra aún en etapas tempranas, es posible que las relaciones carbono:nitrógeno resulten mejores predictores de la descomposición que el contenido de lignina o la relación lignina: nitrógeno (Taylor et al. 1989). De acuerdo a este postulado y a lo discutido anteriormente, en el presente trabajo encontramos que las relaciones fibras:nitrógeno y holocelulosa: nitrógeno fueron las variables que mejor se asociaron a la descomposición de la broza en los dos períodos de incubación considerados. Es decir, y en línea con la mayoría de los estudios citados, los resultados de este trabajo sugieren que las variables de calidad química que predicen de manera más precisa la tasa de descomposición son aquellas que relacionan ambas fracciones, los compuestos lábiles y los recalcitrantes de la broza.

Con respecto a las características de las hojas verdes, varios trabajos han reportado que el contenido de nitrógeno, el área foliar específica y la dureza foliar se asocian con la descomposición de la broza (Garnier et al. 2004; Vaieretti et al. 2005; Cortez et al.2007; Cornwell et al. 2008). En particular, el área foliar específica tiende a estar asociada positivamente al contenido de nitrógeno y negativamente a compuestos recalcitrantes de la broza (e.g., lignina) y, por lo tanto, suele asociarse de manera positiva con la descomposición de las especies (Cornelissen et al. 1999). Sin embargo, en el presente trabajo el área foliar específica no se relacionó significativamente ni a la descomposición de la broza ni a sus compuestos recalcitrantes (celulosa, hemicelulosa, lignina y fibras, resultados no mostrados).

Si bien el área foliar específica y la descomposición pueden relacionarse directamente a través de una área de exposición mayor para los micro-organismos (Swift et al. 1979), éste es un carácter más relacionado a la tasas de crecimiento (Reich et al. 2003; Westoby et al. 2002; Wright et al. 2004; Díaz et al. 2004), que a su vez puede estar relacionado con la descomposición, pero no siempre o no de manera directa como otros caracteres químicos (nitrógeno) y físicos (dureza de la hoja o contenido de materia seca foliar). Los trabajos que predicen una alta descomposición para especies con una gran área foliar se basan en que los atributos químicos (relacionados al uso de nutrientes) y los estructurales (relacionados a la defensa contra herbívoros), que maximizan la taza de fotosíntesis, deberían tenerconsecuenciassobre el ciclado de nutrientes (Santiago et al. 2007). Sin embargo, hay en la literatura evidencia en contra de esta hipótesis. En un trabajo para la flora de Gran Bretaña, Cornelissen et al. (1997) encontraron evidencia que la descomposición es el legado de los caracteres adaptativos relacionados al crecimiento y la defensa de las plantas sólo para monocotiledóneas, ya que las dicotiledóneas herbáceas parecen invertir poco en defensa y el área foliar de las mismas no se relaciona a su descomposición. En otro trabajo de Cornelissen et al. (1999) en el que se incluyen especies de la flora del centro de Argentina, tampoco encontraron una asociación entre el área foliar específica y su descomposición. En este trabajo encontraron que algunas especies, inclusive formas de vida completas, exhibían una combinación de gran área foliar pero gran dureza y baja palatabilidad para herbívoros generalistas al mismo tiempo. Considerando lo expuesto podría decirse que el área foliar específica no sería un carácter confiable para predecir la descomposición de la broza, como ya ha manifestado Wilson et al. (1999) para la flora de Gran Bretaña.

\section{Controles de la descomposición en la flora de la parte alta vs. de la parte baja de las sierras de Córdoba}

Respecto a la comparación de los controles de calidad foliar sobre la descomposición de la broza reportados en el presente estudio y en el realizado para la flora de la parte baja de las sierras de Córdoba, encontramos amplias coincidencias (Tabla 1). El contenido de fibras y la relación fibras:nitrógeno fueron las variables de calidad de la broza que mejor se relacionaron a la descomposición en ambos sistemas y durante los dos períodos de incubación estudiados. El nitrógeno también estuvo estrechamente asociado a la descomposición en ambos sistemas (Tabla 1), aun cuando para las especies de la parte baja de las sierras se trató de nitrógeno medido en hojas verdes y no en la broza como en el presente trabajo. Cabe destacar que el coeficiente de correlación entre el nitrógeno y la descomposición para las especies de las parte baja de las sierras disminuye para el segundo periodo de incubación de la broza respecto a la parte alta de las sierras (Tabla1). Esto puede deberse a que la descomposición estaba más avanzada en dicho experimento ( $80 \%$ con respecto a $42 \%$ en el presente estudio) y quizás el control por parte del nitrógeno pasó a ser menos importante para la 
broza de la parte baja de las sierras (Berg 1986; Palm \& Rowland 1997). En nuestro trabajo, en el que predominaron las graminoides y gramíneas en mata, la holocelulosa (suma de celulosa y hemicelulosa) se correlacionó de forma estrecha con la descomposición de la broza, lo que indica que estos componentes podrían ser importantes en la descomposición de estas especies, en las que su variación es mucho más importante que la de la lignina (Tabla 1). Finalmente, tanto para la flora de la parte baja como para la parte alta de las sierras de Córdoba el área foliar específica de las hojas verdes no se correlacionó significativamente con la pérdida de peso seco de la broza.

En conclusión, el patrón de descomposición de las 24 especies dominantes de los pastizales mésicos y húmedos de la parte alta de las sierras de Córdoba fue consistente entre ambos períodos de incubación, así como la asociación a los caracteres químicos de la broza. La relación entre un carácter recalcitrante y uno lábil (fibras:nitrógeno) fue la variable que más estrechamente se correlacionó con la descomposición inicial e intermedia de la broza, es decir, a los 70 y 196 días de incubación. Tanto en la consistencia en los patrones de descomposición en los diferentes períodos como en su relación con indicadores de calidad de la broza, nuestros resultados son relativamente coincidentes con lo encontrado anteriormente para otras floras. En particular, los controles químicos de la descomposición de la broza de las especies de la parte alta de las sierras de Córdoba coinciden en gran medida con los controles sobre las especies de la parte baja de las sierras de la misma región, a pesar de los distintos regímenes de temperatura y precipitación (8 ${ }^{\circ} \mathrm{C}$ y $130 \mathrm{~mm}$ de diferencia) y a pesar de la distinta composición de formas de vida que caracteriza a las dos áreas.

Agradecimientos: este trabajo fue realizado gracias al financiamiento de CONICET (PIP-112-200801-01458 y PIP-112-200801-01532), Ministerio de Ciencia y Técnica de Córdoba, SECyT (Universidad Nacional de Córdoba), FONCyT e Inter-American Institute for Global Change Research (CRN II 2015 and CRN II 2005; supported by the US National Science Foundation GrantGEO0452325). Agradecemos al Parque Nacional Quebrada del Condorito donde este trabajo fue realizado. Agradecemos especialmente a dos revisores anónimos y a la editora de la revista por sus comentarios que mejoraron notablemente la calidad del manuscrito.

\section{BIBLIOGRAFÍA}

AERTS, R. 1997. Climate, leaf litter chemistry and leaf litter decomposition in terrestrial ecosystem: a triangular relationship. Oikos, 79:439-449.
BERG, B. 1986. Nutrient release from litter and humus in coniferous forest soils-a mini review. Scand. J. For. Res., 1:359-369.

Berg, B; G EквOHM \& C McClaugherty. 1984. Lignin and holocellulose relations during long-term decomposition of some forest litter. Long-term decomposition in a Scots pine forest IV. Can. J. Bot., 62:2540-2550.

BERG, B. 1986. Nutrient release from litter and humus in coniferous forest soils-a mini review. Scand. J. For. Res., 1:359-369.

BERG, B \& R LASKOWSKI. 2006. Methods in studies of organic matter decay. Pp. 292-314 en: Litter decomposition: A guide to carbon and nutrient turnover. Academic Press. Elsevier.

Cabido, M \& A Acosta. 1985. Estudio fitosociológico en bosques de Polylepis australis BITT. ("Tabaquillo") en las Sierras de Córdoba, Argentina. Doc. Phytosoc., 9: 385-400.

Cabido, M; R Breimer \& G Vega. 1987. Plant communities and associated soil types in a high plateau of the Córdoba mountains, central Argentina. Mt. Res. Dev., 7:25-42.

CADISH, G \& KE GILLER. 1997. Driven by nature: a sense of arrival or departure? Capítulo 29. Pp. 393-399 en: Cadish, G \& KE Giller (eds.). Driven by Nature: plant litter quality and decomposition. CAB International.

Chapin, FS III; PA Matson \& HA MoOnley. 2002. Terrestrial decomposition. Capítulo 7. Pp.151-175 en: Principles of terrestrial ecosystem ecology. Springer-Verlag, New York, New York, USA.

Cingolani, AM; M Cabido; D Renison \& V Solís NefFa. 2003. Combined effects of environment and grazing on vegetation structure in Argentine granite grasslands. J. Veg. Sci.,14:223-232.

Cingolani, AM; D Renison; M ZaK \& M Cabido 2004. Mapping vegetation in a heterogeneous mountain using Landsat data: an alternative method to define and classify land-cover units. Rem. Sens. Env., 92:84-97.

Cingolani, AM; M Cabido; DE Gurvich; D Renison \& S DíAz. 2007. Filtering processes in the assembly of plant communities: Are species presence and abundance driven by the same traits? J. Veg. Sci., 18:911-920.

Cingolani, AM; D Renison; PA Tecco; D Gurvich \& M CABIDO. 2008. Predicting cover types in a mountain range with long evolutionary grazing history: a GIS approach. J. Biogeogr., 35:538-551.

Colladon,L; GSFelici \& IPazos. 2010. Anuario Pluviométrico 2005/6-2009/10. Cuenca del Río San Antonio. Sistema del Río Suquia-Provincia de Córdoba. Instituto Nacional del agua y del ambiente (INA) y centro de investigaciones de la Región Semiárida (CIRSA).

Cornelissen, JHC. 1996. An experimental comparison of leaf decomposition rates in a wide range of temperate plant species and types. J. Ecol., 84:573-582.

Cornelissen, JHC \& K Thompson. 1997. Functional leaf attributes predict litter decomposition rate in herbaceous plants. New Phytol., 135:109-114.

Cornelissen, JHC; N Pèrez-Harguindeguy; S Diaz; P Grime B Marzano; et AL. 1999. Leaf structure and defense control litter decomposition rate across species and life forms in regional floras on two continents. New Phytol., 143:191-200.

Cornwell, WK; JHC Cornelissen; K Amatangelo; E DORREPAAL; VT EVINER; ET AL. 2008. Plant species traits are the predominant control on litter decomposition rates within biomes worldwide. Ecol. Lett., 11:1065-1071.

Cortez, J; M Demard; P Bottner \& L Jocteur Monrozier. 
1996. Decomposition of Mediterranean leaf litters: A microcosm experiment investigating relationships between decomposition rates and litter quality. Soil Biol. Biochem., 28:443-452.

Cortez, J; E Garnier; N Pérez Harguindeguy; M Debussche \& D Gillon. 2007. Plant traits, litter quality and decomposition in a Mediterranean old-field succesion. Plant Soil, 296:19-34.

DíAz, S; A ACOSTA \& M CABIDO. 1994. Community structure in montane grasslands of central Argentina in relation to land use. J. Veg. Sci., 5:483-488.

Díaz, S; JG Hodgson; K Thompson; M Cabido; JHC Cornelissen; ET AL. 2004. The plant traits that drive ecosystems: Evidence from three continents. J. Veg. Sci., 15:295-304.

Di RIENZO, JA; F CASANOVES; MG BALZARINI; L GONZÁLEZ; M TABLADA; ET AL. InfoStat versión 2013. Grupo InfoStat, FCA, Universidad Nacional de Córdoba, Argentina. http://www.infostat.com.ar.

Garnier, E; J Cortez; G Billès; ML Navas; C Roumet; et AL. 2004. Plant functional markers capture ecosystem properties during secondary succession. Ecology, 85: 2630-2637.

GoerIng, HK \& PJ VAn Soest. 1970. Forage Fiber Analyses. Handbook $N^{\circ} 379$, Department of Agriculture, USDA, Washington D.C.

Kazakou, E; C Violle; C Roumet; C Pintor; O GimeneZ; et AL. 2009. Litter quality and decomposability of species from a Mediterranean succession depend on leaf traits but not on nitrogen supply. Ann. Bot., 104:1151-1161.

Lavelle, P; E Blanchart; S Martin; AV Spain; F Toutain; ET AL. 1993. A hierarchical model for decomposition in terrestrial ecosystems: applications to soils of the humid tropics. Biotropica, 25:130-150.

Loranger, G; Di Ponce \& P Lavelle. 2002. Leaf decomposition in two semi-evergreen tropical forests: influence of litter quality. Biol. Fertile Soils, 35:247-252.

Melillo, JM; JD Aber \& JF Muratore. 1982. Nitrogen and lignin control of hardwood leaf litter decomposition dynamics. Ecology, 63:621-626.

MEENTEMEYER, V. 1978 Macroclimate and lignin control of litter decomposition rates. Ecology, 59:465-472.

O'NeILL, J \& R Wевв. 1970. Simultaneous determination of nitrogen, phosphorus and potassium in plant material by automatic methods. J. Sci. Food Agr., 21:217-219.

Palm, CA \& AP Rowland. 1997. A minimum dataset for characterization of plant quality for decomposition. Pp. 379-392 en: Cadisch, G \& KE Giller (eds.). Driven by Nature: Plant Litter Quality and Decomposition. CAB International-University Press, Cambridge.

PAKEMAN, RJ \& HM Quested. 2007. Sampling plant functional traits: What proportion of the species need to be measured? Appl. Veg. Sci., 10:91-96.

Pérez Harguindeguy, N; S Díaz; JHC Cornelissen; F Vendramini; M Cabido; et Al. 2000a. Chemistry and toughness predict leaf litter decomposition rates over a wide spectrum of functional types and taxa in central Argentina. Plant Soil, 218:21-30.

Pérez Harguindeguy, N; F Vendramini; S Díaz; M Cabido; JHC Cornelissen; et al. 2000b. Descomposición y caracteres foliares de especies de Pteridófitas y Angiospermas del Chaco Serrano de Córdoba, Argentina. Kurtziana, 28:35-44.
Pérez Harguindeguy, N; S Díaz; E Garnier; S Lavorel; H POORTER; ET AL. 2013. New handbook for standardised measurement of plant functional traits worldwide. Aust. J. Bot., 61:167-234.

Pucheta, E; F Vendramini; M Cabido \& S Díaz. 1998a. Estructura y funcionamiento de un pastizal de montaña bajo pastoreo y su respuesta luego de su exclusión. Rev. Fac. Agr., 103(1):77-92.

Pucheta, E; M Cabido; S Díaz \& G Funes. 1998b. Floristic composition, biomass and aboveground net plant production in grazed and protected sites in a mountain grassland of central Argentina. Acta Oecol., 19:97-105.

Reich, PB; IJ Wright; J Cavender"Bares; JM Craine; J OleKSYN; ET AL. 2003. The evolution of plant functional variation: traits, spectra, and strategies. Int. J. Plant Sci., 164:S143-S164.

SANTIAGO, LS \& SJ WRIGHT. 2007. Leaf functional traits of tropical forest plants in relation to growth form. Funct. Ecol., 21:19-27.

Swift, MJ; OW Heal \& JM Anderson, JM. 1979. Decomposition in terrestrial ecosystems. Studies in Ecology. Blackwell, Oxford, UK.

TAYLOR, BR; D PARKINSON \& WFJ PARSONs. 1989. Nitrogen and lignin content as predictors of litter decay rates: a microcosm test. Ecology, 70:97-104.

Vaieretti, MV; N Pérez Harguindeguy; D Gurvich; AM Cingolani \& M Cabido. 2005. Descomposition dynamis and physico-chemical leaf quality of abundant species in a montane woodland in central Argentina. Plant Soil, 278:223-234.

VAieretTi, MV. 2010. Estructura de la vegetación y dinámica de la descomposición: El rol de la herbivoría en pastizales de altura bajo uso ganadero (Córdoba, Argentina). Tesis Doctoral. Facultad de Ciencias Exactas, Físicas y Naturales Universidad Nacional de Córdoba, Córdoba, Argentina.

Vaieretti, MV; AM Cingolani; N Perez-Harguindeguy; DE Gurvich \& M CABido. 2010. Does decomposition of standard materials differ among grassland patches maintained by livestock? Austral Ecol., 35:935-943.

Vaieretti, MV; AM Cingolani; N Perez Harguindeguy \& M CABIDO. 2013. Effects of differential grazing on decomposition rate and nitrogen availability in a productive mountain grassland. Plant Soil. DOI: 10.1007/s11104-013-1831-9.

VivANCO, L \& AT Austin. 2006. Intrinsic effects of species on leaf litter and root decomposition: a comparison of temperate grasses from North and South America. Oecologia, 150:97-107.

Westoby, M; DS Falster; AT Moles; PA Vesk \& IJ WRIGHT. 2002. Plant ecological strategies: some leading dimensions of variation between species. Annu. Rev. Ecol. Syst., 33:125-159.

Wilson, PJ; K THOMPSON \& JG Hodgson. 1999. Specific leaf area and leaf dry matter content as alternative predictors of plant strategies. New Phytol., 143:155-162.

ZhanG, D; D HuI; Y LuO \& G ZHou. 2008. Rates of litter decomposition in terrestrial ecosystems: global patterns and controlling factors. J. Plant Ecol., 1(2):85-93.

Zuloaga, FO; O Morrone \& MJ Belgrano. 2008. Catálogo de las Plantas Vasculares del Cono Sur (Argentina, Sur de Brasil, Chile, Paraguay y Uruguay). Monogr. Missouri Bot. Garden (USA). 


\section{INFORMACIÓN SUPLEMENTARIA}

Tabla S1. Nombre científico, familia, y forma de vida de las 24 especies seleccionadas dominantes de los pastizales mésicos y húmedos de las sierras altas de Córdoba, Argentina

Table S1. Scientific name, family and life form for the selected 24 dominant species of the mesic and humid grasslands of the highland of Córdoba Mountains, Argentina.

\begin{tabular}{|c|c|c|}
\hline Especie & Familia & Forma de vida \\
\hline Bidens andicola var. decomposita Kunth & Asteraceae & dicotiledónea herbácea \\
\hline Eryngium agavifolium Griseb. & Apiaceae & dicotiledónea herbácea \\
\hline Eryngium nudicaule Lam. & Apiaceae & dicotiledónea herbácea \\
\hline Glandularia dissecta (Willd. ex Spreng.) Schnack \& Covas & Verbenaceae & dicotiledónea herbácea \\
\hline Grindelia globularifolia Griseb. & Asteraceae & dicotiledónea herbácea \\
\hline Lachemilla pinnata (Ruiz \& Pav.) Rothm. & Rosaceae & dicotiledónea herbácea \\
\hline Plantago argentina Pilg. & Plantaginaceae & dicotiledónea herbácea \\
\hline Bromus catharticus var. catharticus Vahl & Poaceae & graminoide \\
\hline Carex fuscula ssp. fuscula d'Urv. & Cyperaceae & graminoide \\
\hline Chascolytrum subaristatum (Lam.) Desv. & Poaceae & graminoide \\
\hline Eleocharis pseudoalbibracteata S. González \& Guagl. & Cyperaceae & graminoide \\
\hline Eragrostis lugens Nees. & Poaceae & graminoide \\
\hline Festuca dissitiflora Steud. ex Griseb. & Poaceae & graminoide \\
\hline Juncus pallescens var. achalensis Lam. & Juncaceae & graminoide \\
\hline Juncus uruguensis Griseb. & Juncaceae & graminoide \\
\hline Muhlenbergia peruviana (P. Beauv.) Steud. & Poaceae & graminoide \\
\hline Poa hubbardiana Parodi & Poaceae & graminoide \\
\hline Sisyrinchium unguiculatum Griseb. & Iridaceae & graminoide \\
\hline Sorghastrum pellitum (Hack.) Parodi & Poaceae & graminoide \\
\hline Sporobolus indicus (L.) R. Br. var. indicus & Poaceae & graminoide \\
\hline Deyeuxia hieronymi (Hack.) Türpe & Poaceae & gramínea en mata \\
\hline Festuca lilloi Hack. & Poaceae & gramínea en mata \\
\hline Nassella nidulans (Mez) Barkworth & Poaceae & gramínea en mata \\
\hline Poa stuckertii (Hack.) Parodi & Poaceae & gramínea en mata \\
\hline
\end{tabular}

Nota: la forma de vida graminoide incluye a gramíneas bajas y otras monocotiledóneas graminiformes 
Tabla S2. Valores medios y errores estándar del contenido de celulosa, hemicelulosa, lignina y nitrógeno de la broza y valores medios del área foliar específica de las hojas verdes de las 24 especies dominantes de los pastizales mésicos y húmedos de sierras altas de Córdoba, Argentina.

Table S2. Mean values and standard errors of litter's cellulose, hemicelluloses, lignin, and nitrogen content, and mean values of specific leaf area of the green leaves of 24 dominant species of the mesic and humid grasslands of the highland of Córdoba Mountains, Argentina.

\begin{tabular}{|c|c|c|c|c|c|c|}
\hline Especie & $\begin{array}{l}\text { Forma } \\
\text { de vida }\end{array}$ & $\begin{array}{c}\text { Celulosa } \\
(\%)\end{array}$ & $\begin{array}{l}\text { Hemicelulosa } \\
(\%)\end{array}$ & $\underset{(\%)}{\text { Lignina }}$ & $\begin{array}{c}\text { Nitrógeno } \\
(\%)\end{array}$ & $\begin{array}{l}\text { Área foliar } \\
\text { específica } \\
\left(\mathrm{mm}^{2} / \mathrm{mg}\right)\end{array}$ \\
\hline Bidens andicola & $\mathrm{d}$ & $20.7 \pm 0.9$ & $0.0 \pm 0.0$ & $18.6 \pm 0.6$ & $2.1 \pm 0.2$ & $20^{\S}$ \\
\hline Eryngium agavifolium & d & $37.5 \pm 0.8$ & $2.7 \pm 1.4$ & $9.2 \pm 0.2$ & $0.8 \pm 0.1$ & $6^{\S}$ \\
\hline Eryngium nudicaule & $\mathrm{d}$ & $28.5 \pm 0.4$ & $0.0 \pm 0.0$ & $8.9 \pm 0.7$ & $1.2 \pm 0.1$ & $7^{\S}$ \\
\hline Glandularia dissecta & $\mathrm{d}$ & $21.6 \pm 0.3$ & $0.0 \pm 0.0$ & $18.8 \pm 0.5$ & $1.6 \pm 0.1$ & 20 \\
\hline Grindelia globularifolia & $\mathrm{d}$ & $20.9 \pm 1.1$ & $0.0 \pm 0.0$ & $10.7 \pm 1.0$ & $1.2 \pm 0.0$ & 9 \\
\hline Lachemilla pinnata & $\mathrm{d}$ & $28.4 \pm 0.8$ & $3.8 \pm 0.3$ & $9.9 \pm 0.03$ & $1.1 \pm 0.1$ & $14^{\S}$ \\
\hline Plantago argentina & $\mathrm{d}$ & $28.9 \pm 0.6$ & $0.0 \pm 0.0$ & $19.2 \pm 0.8$ & $1.0 \pm 0.1$ & 7 \\
\hline Bromus catharticus & g & $44.9 \pm 0.3$ & $20.9 \pm 0.1$ & $5.3 \pm 0.1$ & $0.7 \pm 0.1$ & $18^{\S}$ \\
\hline Carex fuscula & $\mathrm{g}$ & $31.3 \pm 0.4$ & $25.1 \pm 1.1$ & $9.8 \pm 0.4$ & $0.8 \pm 0.2$ & $18^{\S}$ \\
\hline Chascolytrum subaristatum & $\mathrm{g}$ & $42.3 \pm 1.0$ & $22.3 \pm 0.8$ & $6.1 \pm 0.4$ & $0.8 \pm 0.1$ & $12^{\S}$ \\
\hline Eleocharis pseudoalbibracteata & $\mathrm{g}$ & $38.5 \pm 0.3$ & $18.1 \pm 2.5$ & $8.4 \pm 0.2$ & $1.4 \pm 0.1$ & $27 \AA$ \\
\hline Eragrostis lugens & $\mathrm{g}$ & $34.7 \pm 0.3$ & $25.0 \pm 1.2$ & $5.3 \pm 0.1$ & $1.0 \pm 0.1$ & $8^{\S}$ \\
\hline Festuca dissitiflora & $\mathrm{g}$ & $38.4 \pm 0.6$ & $24.4 \pm 1.6$ & $4.4 \pm 0.1$ & $0.7 \pm 0.1$ & 12 \\
\hline Juncus pallescens & $\mathrm{g}$ & $37.1 \pm 0.7$ & $19.6 \pm 1.9$ & $10.2 \pm 1.0$ & $1.6 \pm 0.0$ & $7 \S$ \\
\hline Juncus uruguensis & $\mathrm{g}$ & $35.4 \pm 0.5$ & $29.5 \pm 0.3$ & $6.7 \pm 0.3$ & $1.4 \pm 0.1$ & $8^{\S}$ \\
\hline Muhlenbergia peruviana & $\mathrm{g}$ & $33.5 \pm 0.8$ & $34.4 \pm 3.3$ & $4.7 \pm 0.3$ & $0.9 \pm 0.1$ & $14^{\S}$ \\
\hline Poa hubbardiana & $\mathrm{g}$ & $42.5 \pm 2.2$ & $23.5 \pm 1.7$ & $5.2 \pm 0.6$ & $0.6 \pm 0.0$ & $21^{\S}$ \\
\hline Sisyrinchium unguiculatum & $\mathrm{g}$ & $35.4 \pm 0.1$ & $5.6 \pm 0.8$ & $11.8 \pm 0.4$ & $1.2 \pm 0.0$ & $9^{\S}$ \\
\hline Sorghastrum pellitum & $\mathrm{g}$ & $37.9 \pm 1.0$ & $19.8 \pm 0.0$ & $7.6 \pm 0.4$ & $0.8 \pm 0.1$ & $7 \S$ \\
\hline Sporobolus indicus & $\mathrm{g}$ & $34.7 \pm 1.0$ & $31.8 \pm 1.3$ & $3.8 \pm 0.0$ & $1.2 \pm 0.1$ & $10^{\S}$ \\
\hline Deyeuxia hieronymi & gm & $39.7 \pm 1.8$ & $26.9 \pm 1.2$ & $7.2 \pm 0.3$ & $0.5 \pm 0.1$ & $10^{\S}$ \\
\hline Festuca lilloi & gm & $47.9 \pm 0.7$ & $18.8 \pm 1.4$ & $7.7 \pm 0.6$ & $0.5 \pm 0.1$ & $6^{\S}$ \\
\hline Nassella nidulans & gm & $41.0 \pm 1.3$ & $20.5 \pm 1.1$ & $6.5 \pm 0.3$ & $0.7 \pm 0.1$ & 12 \\
\hline Poa stuckertii & gm & $44.4 \pm 1.0$ & $23.8 \pm 0.8$ & $7.7 \pm 0.3$ & $0.4 \pm 0.0$ & $6^{\S}$ \\
\hline
\end{tabular}

Notas. d: dicotiledónea; g: graminoide; gm: gramínea en mata. § datos obtenidos de Díaz et al. 2004 y Cingolani et al. 2007 
Tabla S3. Valores medios y errores estándar del porcentaje de holocelulosa (celulosa+hemicelulosa) y fibras (celulo sa+hemicelulosa+lignina), de la proporción holocelulosa/fibras y de las relaciones lignina:nitrógeno, holocelulosa: nitrógeno y fibras:nitrógeno de la broza de las 24 especies dominantes de los pastizales mésicos y húmedos de las sierras altas de Córdoba, Argentina.

Table S3. Mean values and standard errors of the percentage of holocellulose (cellulose+hemicellulose) and fiber (celulose+hemicellulose+lignin), the proportion of holocellulose / fibers and the lignin:nitrogen, holocellulose:nitrogen and fiber:nitrogen ratios of the litter of 24 dominant species of the mesic and humid grasslands of the highland of Córdoba Mountains, Argentina.

\begin{tabular}{|c|c|c|c|c|c|c|c|}
\hline Especie & $\begin{array}{l}\text { Forma } \\
\text { de vida }\end{array}$ & Holocelulosa & Fibras & $\begin{array}{l}\text { Holocelulosa / } \\
\text { fibras }\end{array}$ & $\begin{array}{l}\text { Lignina: } \\
\text { nitrógeno }\end{array}$ & $\begin{array}{l}\text { Holocelulosa: } \\
\text { nitrógeno }\end{array}$ & $\begin{array}{c}\text { Fibras: } \\
\text { nitrógeno }\end{array}$ \\
\hline Bidens andicola & $\mathrm{d}$ & $20.7 \pm 0.9$ & $39.3 \pm 0.4$ & $0.5 \pm 0.02$ & $9.2 \pm 0.8$ & $10.4 \pm 1.7$ & $19.5 \pm 2.6$ \\
\hline Eryngium agavifolium & d & $40.2 \pm 1.6$ & $49.4 \pm 1.5$ & $0.8 \pm 0.0008$ & $12.4 \pm 1.1$ & $54.3 \pm 5.9$ & $66.7 \pm 6.9$ \\
\hline Eryngium nudicaule & $\mathrm{d}$ & $28.5 \pm 0.4$ & $37.4 \pm 0.4$ & $0.8 \pm 0.007$ & $7.6 \pm 0.2$ & $24.6 \pm 1.7$ & $32.2 \pm 1.5$ \\
\hline Glandularia dissecta & $\mathrm{d}$ & $21.6 \pm 0.3$ & $40.4 \pm 0.5$ & $0.5 \pm 0.005$ & $11.7 \pm 0.7$ & $13.4 \pm 0.4$ & $25.1 \pm 1.1$ \\
\hline Grindelia globularifolia & $\mathrm{d}$ & $20.9 \pm 1.1$ & $31.6 \pm 1.3$ & $0.7 \pm 0.004$ & $9.3 \pm 0.5$ & $18.3 \pm 1.3$ & $27.6 \pm 1.0$ \\
\hline Lachemilla pinnata & $\mathrm{d}$ & $32.3 \pm 1.2$ & $42.0 \pm 1.1$ & $0.8 \pm 0.005$ & $9.2 \pm 0.6$ & $30.0 \pm 2.7$ & $39.1 \pm 2.7$ \\
\hline Plantago argentina & d & $28.9 \pm 0.6$ & $48.1 \pm 1.3$ & $0.6 \pm 0.002$ & $19.2 \pm 0.8$ & $28.9 \pm 1.3$ & $48.1 \pm 1.9$ \\
\hline Bromus catharticus & $\mathrm{g}$ & $65.9 \pm 0.3$ & $71.1 \pm 0.3$ & $0.9 \pm 0.008$ & $7.7 \pm 0.6$ & $97.1 \pm 7.5$ & $104.8 \pm 8.1$ \\
\hline Carex fuscula & $\mathrm{g}$ & $56.4 \pm 1.0$ & $66.2 \pm 0.6$ & $0.8 \pm 0.02$ & $13.4 \pm 2.5$ & $75.9 \pm 10.8$ & $89.4 \pm 13.3$ \\
\hline Chascolytrum subaristatum & $\mathrm{g}$ & $64.6 \pm 0.9$ & $70.7 \pm 1.0$ & $0.9 \pm 0.002$ & $7.6 \pm 0.7$ & $80.7 \pm 7.0$ & $88.3 \pm 7.5$ \\
\hline Eleocharis pseudoalbibracteata & $\mathrm{g}$ & $56.6 \pm 2.2$ & $65.0 \pm 2.1$ & $0.9 \pm 0.008$ & $6.0 \pm 0.1$ & $40.4 \pm 1.5$ & $46.4 \pm 1.4$ \\
\hline Eragrostis lugens & $\mathrm{g}$ & $59.7 \pm 0.9$ & $65.0 \pm 0.8$ & $0.9 \pm 0.009$ & $5.2 \pm 0.4$ & $58.5 \pm 3.1$ & $63.7 \pm 3.5$ \\
\hline Festuca dissiti ora & $\mathrm{g}$ & $62.8 \pm 2.2$ & $67.2 \pm 2.3$ & $0.9 \pm 0.03$ & $6.4 \pm 0.5$ & $91.7 \pm 9.2$ & $98.1 \pm 9.7$ \\
\hline Juncus pallescens & $\mathrm{g}$ & $56.7 \pm 1.3$ & $66.9 \pm 0.3$ & $0.9 \pm 0.02$ & $6.5 \pm 0.9$ & $36.2 \pm 0.4$ & $42.8 \pm 1.2$ \\
\hline Juncus uruguensis & $\mathrm{g}$ & $64.9 \pm 0.8$ & $71.6 \pm 0.8$ & $0.9 \pm 0.003$ & $4.9 \pm 0.5$ & $47.5 \pm 3.0$ & $52.5 \pm 3.4$ \\
\hline Muhlenbergia peruviana & $\mathrm{g}$ & $67.9 \pm 3.8$ & $72.6 \pm 3.9$ & $0.9 \pm 0.007$ & $5.8 \pm 1.0$ & $83.9 \pm 15.7$ & $89.7 \pm 16.7$ \\
\hline Poa hubbardiana & $\mathrm{g}$ & $65.9 \pm 0.9$ & $71.1 \pm 0.4$ & $0.9 \pm 0.004$ & $8.6 \pm 0.8$ & $110.9 \pm 5.1$ & $119.5 \pm 4.6$ \\
\hline Sisyrinchium unguiculatum & $\mathrm{g}$ & $41.0 \pm 0.8$ & $52.8 \pm 1.0$ & $0.8 \pm 0.005$ & $10.1 \pm 0.3$ & $35.0 \pm 0.5$ & $45.0 \pm 0.7$ \\
\hline Sorghastrum pellitum & $\mathrm{g}$ & $57.7 \pm 1.0$ & $65.4 \pm 0.6$ & $0.9 \pm 0.008$ & $9.6 \pm 0.4$ & $73.3 \pm 7.3$ & $82.9 \pm 7.6$ \\
\hline Sporobolus indicus & $\mathrm{g}$ & $66.5 \pm 0.8$ & $70.3 \pm 0.8$ & $0.9 \pm 0.009$ & $3.2 \pm 0.2$ & $57.0 \pm 3.3$ & $60.2 \pm 3.5$ \\
\hline Deyeuxia hieronymi & gm & $66.6 \pm 0.8$ & $73.8 \pm 0.5$ & $0.9 \pm 0.004$ & $14.5 \pm 2.0$ & $136.3 \pm 24.3$ & $150.7 \pm 26.3$ \\
\hline Festuca lilloi & gm & $66.7 \pm 1.8$ & $74.5 \pm 1.7$ & $0.9 \pm 0.005$ & $14.8 \pm 2.7$ & $126.4 \pm 14.6$ & $141.3 \pm 17$ \\
\hline Nassella nidulans & gm & $61.5 \pm 0.8$ & $68.0 \pm 0.5$ & $0.9 \pm 0.007$ & $9.5 \pm 1.5$ & $91.3 \pm 16.5$ & $100.8 \pm 17.9$ \\
\hline Poa stuckertii & gm & $68.2 \pm 0.6$ & $75.9 \pm 0.8$ & $0.9 \pm 0.0001$ & $18.3 \pm 1.0$ & $163.3 \pm 5.8$ & $181.7 \pm 6.5$ \\
\hline
\end{tabular}

Notas. d: dicotiledónea; g: graminoide; gm: gramínea en mata 